\title{
Correction to: Matrix spectral factorization for SA4 multiwavelet
}

\author{
Vasil Kolev ${ }^{1} \cdot$ Todor Cooklev $^{2} \cdot$ Fritz Keinert $^{3}$ \\ Published online: 16 October 2018 \\ ○) Springer Science+Business Media, LLC, part of Springer Nature 2018
}

\section{Correction to: Multidim Syst Sign Process (2018) 29:1613-1641 https://doi.org/10.1007/s11045-017-0520-x}

After a careful review of the published version of our paper, we discovered that some references to the number of multiwavelet decomposition and reconstruction levels are incorrect. The pre- and post-processing steps were counted as one level, so the actual number of levels is one less than that described in the original paper.

This affects the labeling and captions of Figs. 9b, 11, and 12, as well as some short passages in the text. The corrected figures and text are below.

\section{Section 5.2.1, last two paragraphs:}

For the test signal 'LoSine', both noisy and noiseless MAEs show a linear increase up to the second level, with only a small increase at the third and fourth levels of $-5.7 \times 10^{-3}$ and $3.6 \times 10^{-3}$, as shown in Fig. 9b. Therefore, after the second level, the influence of noise is weak.

For the test signal 'Piece-regular', the MAE grows quadratically with increasing $j$. The difference between noisy and noiseless signals is smallest at the pre-/post-processing step $\left(6 \times 10^{-5}\right)$ and largest at the second level $\left(1.2 \times 10^{-3}\right)$. For the test signal 'Piece-polynomial', the MAE grows non-uniformly and nonlinearly with increasing $j$. The difference between noisy and noiseless signals is smallest at the pre-/post-processing step $\left(3.8 \times 10^{-4}\right)$ and largest at the third level $\left(2 \times 10^{-3}\right)$.

The original article can be found online at https://doi.org/10.1007/s11045-017-0520-x.

\footnotetext{
$凶$ Vasil Kolev

kolev_acad@abv.bg

Todor Cooklev

cooklevt@pfw.edu

Fritz Keinert

keinert@iastate.edu

1 Institute of Information and Communication Technologies, Bulgarian Academy of Sciences, B1. 2 Acad. G. Bonchev St., 1113 Sofia, Bulgaria

2 Wireless Technology Center, Purdue University, Fort Wayne, IN 46805, USA

3 Department of Mathematics, Iowa State University, Ames, IA 50011, USA
} 
(a)
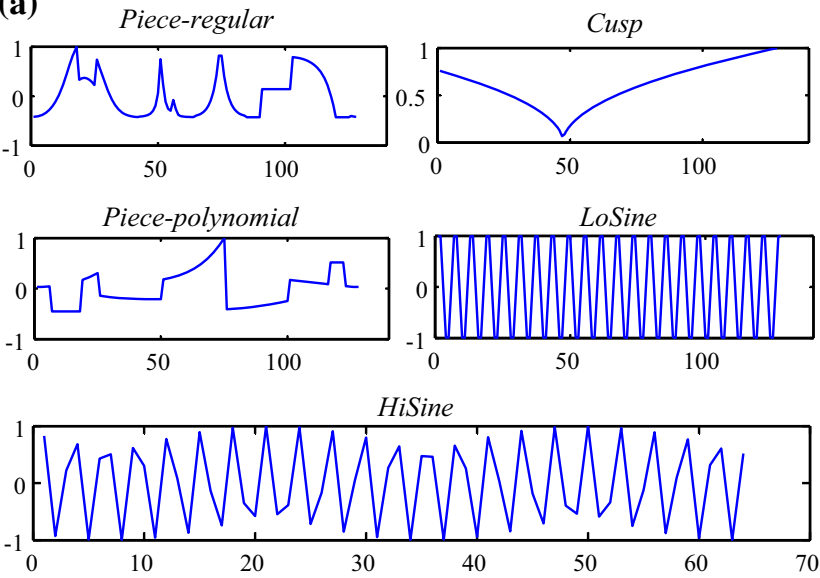

(b)

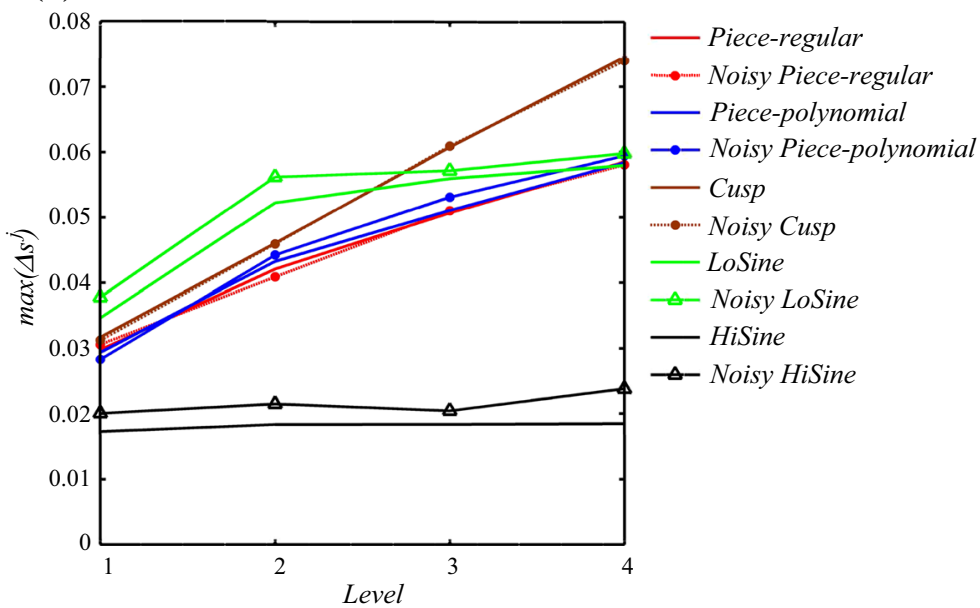

Fig. 9 Decomposition and reconstruction with the balanced approximate SA4 multiwavelet; a The normed test signals; $\mathbf{b}$ the MAEs obtained at level $j$

\section{Section 5.3:}

In this section, we compare the balanced and non-balanced version of the exact SA4 multiwavelet with the GHM multiwavelet (Downie and Silverman 1998) and the Chui-Lian multiwavelet CL (Downie and Silverman 1998), by considering image denoising with vector hard thresholding (Donoho and Johnstone 1994), using 1-5 levels. The images are 'Lena', 'Zelda', and 'House', of size $512 \times 512$ pixels, with white additive Gaussian noise with variance $\sigma=10$. See Fig. 10.

The multiwavelet coefficients of the white noise are reduced at each level, but uniformly distributed within each level. Therefore, the best approach to denoising is to find an appropriate threshold value at each level.

The exact SA4 multiwavelet, both balanced and non-balanced, achieved the maximal PSNRs. The PSNR differences for the both versions of the test images are $3.57 \mathrm{~dB}$ at the pre/post-processing step and $4.06 \mathrm{~dB}$ at the fifth level for "Lena"; $4.17 \mathrm{~dB}$ (pre-/post-processing 
Fig. 11 PSNRs of four images for $2 \mathrm{D}$ decomposition and reconstruction without additional processing of 1 through 5 or 6 levels with the approximate SA4 multiwavelet
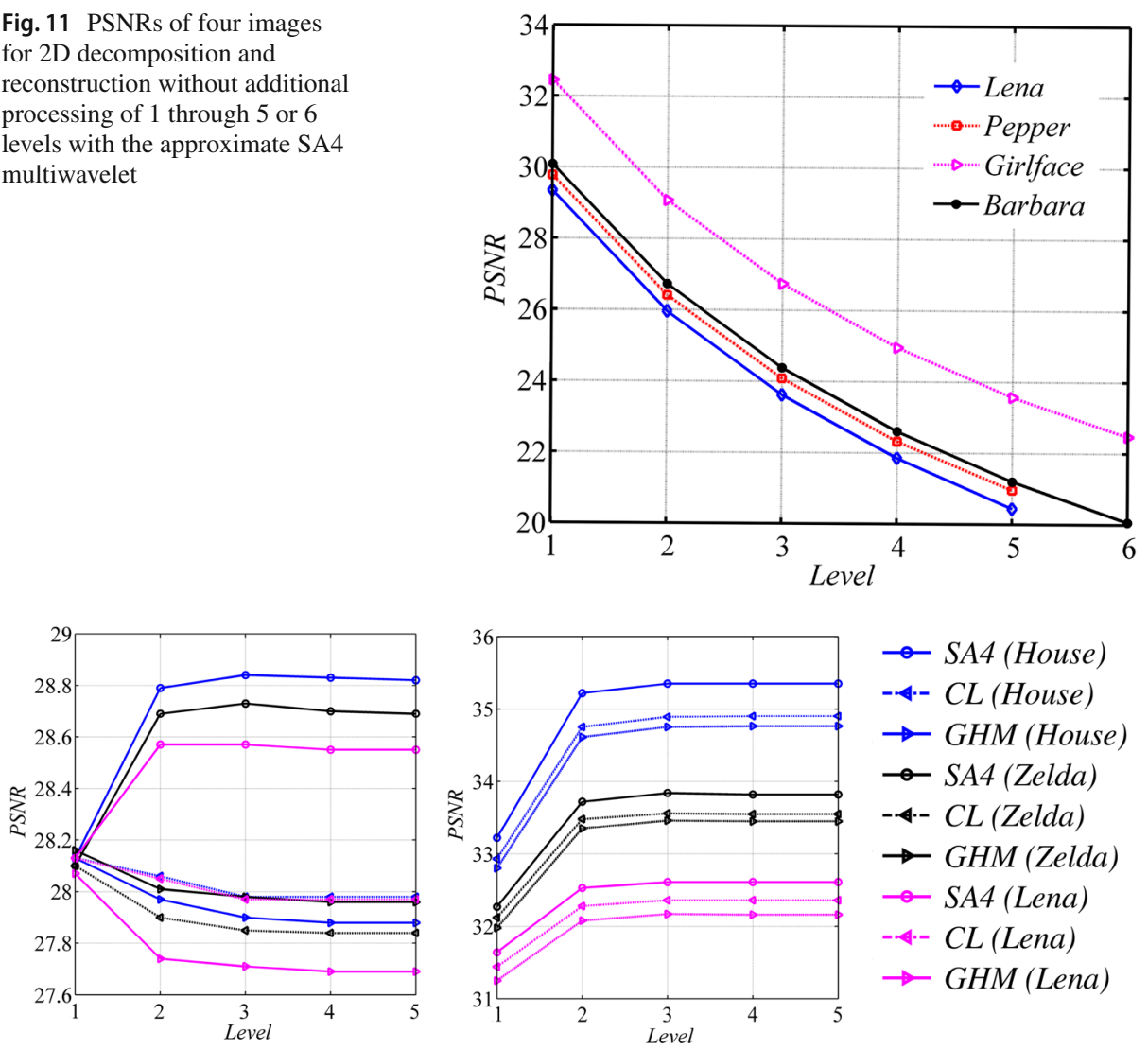

$\rightarrow$ SA4 (House)

-4- CL (House)

$\rightarrow G H M$ (House)

$\rightarrow$ SA4 (Zelda)

-4-- CL (Zelda)

$\rightarrow-G H M($ Zelda $)$

$\rightarrow$ SA4 (Lena)

-4- CL (Lena)

$\rightarrow G H M($ Lena)

Fig. 12 Comparative analysis of PSNRs for image denoising with the exact SA4 multiwavelet and vector hard threshold through 1-5 levels of the images 'House', 'Zelda', and 'Lena' with $512 \times 512$ pixels and white additive Gaussian noise with variance $\sigma=10$. Left: Non-balanced multiwavelets; Right: Balanced multiwavelets

step) and $5.13 \mathrm{~dB}$ (fifth level) for “Zelda”, $5.09 \mathrm{~dB}$ (pre-/post-processing step) and $6.53 \mathrm{~dB}$ (fifth level) for "House". Although balancing of multiwavelets destroys the symmetry, it leads to increasing PSNRs and better image denoising with the exact SA4 multiwavelet for the three test images for the both version multiwavelets, while for the non-balanced GHM and CL multiwavelets, PSNRs decrease (see Fig. 12).

According to PSNRs, image decomposition and reconstruction through two levels with the approximate SA4 multiwavelet is comparable to image denoising with the non-balanced SA4 multiwavelet through three levels, while one level is comparable with the balanced multiwavelet pre-/post-processing step (see Figs. 11, 12).

Publisher's Note Springer Nature remains neutral with regard to jurisdictional claims in published maps and institutional affiliations. 